COLABORACIÓN ESPECIAL

\title{
BROTES EPIDÉMICOS DE SARAMPIÓN EN ANDALUCÍA DURANTE EL PERÍODO 2010-2015
}

\author{
Carmen Montaño Remacha (1), Virtudes Gallardo García (1), M Mar Mochón Ochoa \\ (2), Marcelino García Fernández (3), José María Mayoral Cortés (1) y Josefa Ruiz \\ Fernández (1).
}

(1) Servicio de Epidemiología. Secretaria General de Salud Pública y Consumo. Consejería de Salud. Junta de Andalucía.

(2) Servicio de Epidemiología. Delegación Territorial de Salud de Granada. Consejería de Salud. Junta de Andalucía.

(3) Servicio de Epidemiología. Delegación Territorial de Salud de Sevilla. Consejería de Salud. Junta de Andalucía.

\section{RESUMEN}

El Sistema de Vigilancia Epidemiológica de Andalucía (SVEA) es e encargado de vigilar e investigar urgentemente cualquier notificación de sospecha de sarampión y otras enfermedades de declaración obligatoria y alertas. El objetivo del artículo es describir las características epidemiológicas de los brotes de sarampión ocurridos en Andalucía en los últimos cinco años (2010-2015) así como las medidas de actuación llevadas a cabo durante los mismos. En el periodo estudiado se notificaron tres brotes. El primero de ellos comenzó en Granada en 2010 en población contraria a la vacunación. Las medidas de control adoptadas fueron las recogidas en el protocolo de sarampión del SVEA (aislamiento de casos, identificación e inmunización de contactos), entre las que se incluyeron acciones judiciales ante la negativa de un determinado colectivo a vacunar a sus hijos. El segundo brote comenzó en Sevilla en 2011 en una de las zonas denominadas "con necesidades de transformación social" y se extendió por toda la provincia. A partir de aquel brote se introdujo de forma rutinaria la revisión de las coberturas vacunales dentro del sistema de vigilancia, identificando a los grupos más vulnerables. Durante el primer semestre de 2015 ha habido un pequeño brote en Granada con 15 casos. Se controló rápidamente debido a la actuación precoz en la mayoría de los casos, a la instauración de las medidas correspondientes en centros sanitarios y escolares y a la alta cobertura vacunal de la población.

Palabras clave: Brotes epidémicos. Sistema de información. Sarampión. Vacunación masiva. Vacuna contra el sarampión.

Correspondencia

Carmen Montaño Remacha

Servicio de Epidemiología y Salud Laboral

Consejería de Salud., Junta de Andalucía

Avenida de la Innovación s/n, Edificio Arena, 1

41020 Sevilla

mariac.montano.sspa@juntadeandalucia.es

DOI:

\section{ABSTRACT \\ Outbreaks of Measles in Andalusia, Spain, during the Period 2010-2015}

The Andalusian Surveillance Epidemiological System (SVEA) controls and investigates any notification of measles or any other communicable disease. The aim of this article is to describe the epidemiological characteristics of measles outbreaks occurred in Andalusia in the last five years (2010-2015) and their control measures. In this period three outbreaks were reported: the first one started in Granada in 2010 in a community of objectors to vaccination. Control measures of measles protocol of SVEA were adopted (case isolation, identification and contacts immunization), including judicial measures among the group who refused the vaccination. The second outbreak started in Seville in 2011 in an "area in need of social transformation" and it spread throughout the region. The routine vaccination coverage review was introduced within the surveillance system after those outbreaks, identifying the most vulnerable people. During the first six months of 2015 , a small outbreak, of 15 cases, occurred in Granada. The outbreak was controlled mainly due to the early intervention, the health measures adopted in the schools and health centres involved and the high vaccination coverage achieved in the population.

Keywords: Outbreaks. Information system. Measles. Mass vaccination. Measles vaccine. 


\section{INTRODUCCIÓN}

El Sistema de Vigilancia Epidemiológica de Andalucía (SVEA) se creó en $1996^{1}$ con la finalidad de prevenir la enfermedad mediante medidas de control individuales o colectivas, de forma inmediata o a medio y largo plazo, identificando los problemas de salud y sus factores determinantes, conociendo su distribución, tendencias y características de la población afectada. El SVEA está estructurado en los siguientes niveles organizativos: Secretaría General de Salud Pública de la Consejería de Salud, Delegaciones Territoriales Provinciales, Distritos de Atención Primaria y Hospitales y se encuentra regulado en la Orden de 19 de diciembre de $1996^{2}$. Según la Orden 11 de diciembre de $2008^{3}$, en la que se establece la relación de Enfermedades de Declaración Obligatoria, toda sospecha de sarampión se considera alerta en salud pública y se debe investigar y comunicar de manera urgente al SVEA. En días laborables, de 8:00 a 15:00 horas si la detección de la alerta se produce en atención primaria (AP) la notificación se efectúa a Epidemiología de Atención Primaria (EAP), mediante teléfono. Si se produce en atención hospitalaria se realizará a Medicina Preventiva (MP), que contacta telefónicamente con EAP correspondiente, quien es el encargado de ponerse en contacto con el Centro de Salud implicado para coordinar las medidas correspondientes. Bien EAP o bien MP graban el registro correspondiente en la aplicación informática del SVEA (llamada RedAlerta) e informan a la Delegación Provincial de Salud, Sección de Epidemiología. Entre las 15:00 y las 8:00 horas del día siguiente, los fines de semana y los festivos la comunicación se realizará a la Empresa Pública de Emergencias Sanitarias (EPES), de la Consejería de Salud, la cual avisa telefónicamente a la Red de Alerta del Sistema Sanitario Público de Andalucía ${ }^{4}$, servicio que detecta e interviene de manera urgente ante situaciones de riesgo para la salud pública, potenciales, reales o que generen alarma social.
El protocolo de sarampión del $\mathrm{SVEA}^{5}$ forma parte del Plan de Eliminación del Sarampión en Andalucía ${ }^{6}$ que, al igual que en el resto de España ${ }^{7}$, se puso en marcha en 2001 y que se ajusta a las recomendaciones para la vigilancia del sarampión, la rubéola y el síndrome de rubéola congénita de la Región Europea de la $\mathrm{OMS}^{8}$. Todo caso sospechoso de sarampión ha de ser investigado mediante un protocolo ${ }^{5}$ en menos de 48 horas después de ser notificado.

Respecto a la vacunación del sarampión, fue en 1979 cuando se introdujo la vacuna monovalente en el calendario de vacunación. En 1984 se inició la vacunación con la vacuna triple vírica (TV) (sarampión, rubéola y parotiditis) con una dosis a los 15 meses y una segunda dosis a los 11 años. Se mantuvo así hasta 1999 cuando se adelantó la segunda dosis a los 6 años de edad y en 2004 a los 3 años. Fue en 2012 cuando se adelantó la primera dosis a los 12 meses, manteniéndose así en el momento actual ${ }^{9}$.

Actualmente la cobertura vacunal en Andalucía para la primera y la segunda dosis de TV es superior al $95 \%$ para niños de 1a 2 años y niños de 3 a 6 años respectivamente ${ }^{10}$, porcentaje recomendado por la $\mathrm{OMS}^{11}$ para los países en vías de eliminación de la enfermedad.

Hasta finales de la década de 1980, el patrón epidemiológico del sarampión presentaba ondas epidémicas cada 2 o 3 años. En el momento que la cobertura de vacunación con triple vírica empezó a ser importante (finales de los 80) se rompió este patrón apareciendo ondas epidémicas más distanciadas y con menores tasas de incidencia. En Andalucía, en 1983 se alcanzó la tasa más alta (1.490 casos por 100.000 habitantes) y en 1986 se produjo el siguiente pico epidémico, con una tasa de incidencia de 1.007 casos por 100.000 habitantes, considerándose la última gran epidemia de sarampión en Andalucía. Posteriormente hubo una tendencia decreciente en la tasa de incidencia de la enfermedad que se rompió en 1989, siguiente año epidémico, 
pero ya con una tasa de 125 casos, y en 1995 , último año epidémico, hubo una tasa de incidencia de 39 casos por 100.000 habitantes. Desde 1997 y hasta 2010 las tasas de incidencia de la enfermedad alcanzaron valores muy bajos, excepto en 2003 debido al brote epidémico ocurrido en la provincia de Almería (tasa de incidencia de 2,51 casos por 100.000 habitantes) y en 2008 (con 3,00 casos por 100.000 habitantes) como consecuencia del brote ocurrido en el Distrito Campo de Gibraltar.

El objetivo del artículo es describir las características epidemiológicas de los brotes de sarampión ocurridos en Andalucía en los últimos cinco años y las medidas de actuación llevadas a cabo en ellos.

\section{MATERIAL Y MÉTODOS}

Se analizaron las características epidemiológicas de los últimos brotes de sarampión notificados al SVEA según la descripción de tiempo, lugar y persona y las medidas de control. El período de tiempo estudiado fue desde el 30 de junio de 2010 hasta el 30 de junio de 2015.

La fuente de datos utilizada fue RedAlerta, base electrónica de datos de enfermedades de declaración obligatoria en Andalucía.

La estrategia de vigilancia y control del sarampión en Andalucía está recogida en el protocolo de sarampión ${ }^{5}$ del SVEA, en la que se recogen el modo y circuito de notificación, el formulario de recogida de datos clínicos y epidemiológicos, el tipo de muestras clínicas necesarias, las medidas de control y la clasificación del caso según el origen y el grado de certeza diagnóstica. La definición que se usa de caso sospechoso es muy sensible con el fin de garantizar que los casos no detectados sean mínimos (temperatura corporal superior a $38^{\circ} \mathrm{C}$ y exantema maculopapular con al menos uno de los siguientes tres síntomas: tos, rinitis/coriza y conjuntivitis). Como caso probable se considera a toda persona que cumpla los criterios clínicos y que tenga vín- culo epidemiológico con un caso confirmado por laboratorio y caso confirmado es el de es aquella persona no vacunada recientemente que satisfaga los criterios clínicos y de laboratorio (al menos uno de los cuatro siguientes: respuesta de anticuerpos específicos del virus del sarampión (IgM o seroconversión de $\operatorname{IgG}$ ) en el suero o la saliva; detección de ácido nucleico del virus del sarampión en una muestra clínica; aislamiento del virus del sarampión en una muestra clínica; detección de antígeno del virus del sarampión en una muestra clínica mediante tinción directa con anticuerpos monoclonales fluorescentes específicos del sarampión). En la encuesta epidemiológica se recoge también información sobre el estudio de los contactos (personas que han estado en contacto con un caso de sarampión durante los cuatro días anteriores y los cuatro posteriores a la presentación del exantema). De la investigación de los contactos se detectan casos inmunes, bien por haber padecido la enfermedad o a causa de vacunación correcta, y/o casos susceptibles, sobre los que hay que tomar las medidas de control oportunas, como aislamiento y/o vacunación.

\section{RESULTADOS}

En los últimos cinco años en Andalucía se notificaron dos grandes brotes poblacionales de sarampión, en 2010 y 2011 (figura 1) que afectaron sobre todo a Granada y Sevilla respectivamente. Un pequeño brote fue notificado también en Granada durante el primer semestre de 2015.

Tras los brotes de 2010 y 2011 el número de casos notificados bajó considerablemente. En el año 2012 se declararon 21 casos de sarampión y una tasa de incidencia 0,25 casos por 100.000 habitantes. Fueron casos aislados y 2 pequeños brotes con cuatro personas afectadas cada uno (importados de Madrid y Reino Unido respectivamente). En 2013 se declararon 2 casos confirmados de sarampión, uno aislado y otro relacionado con un brote epidémico en Barcelona, ambos en adultos no vacunados. En 2014 se notificaron 25 casos sospechosos de sarampión, de los que se descartaron 23, 
Figura 1

Casos de sarampión en Andalucía, Junio 2010-Junio 2015

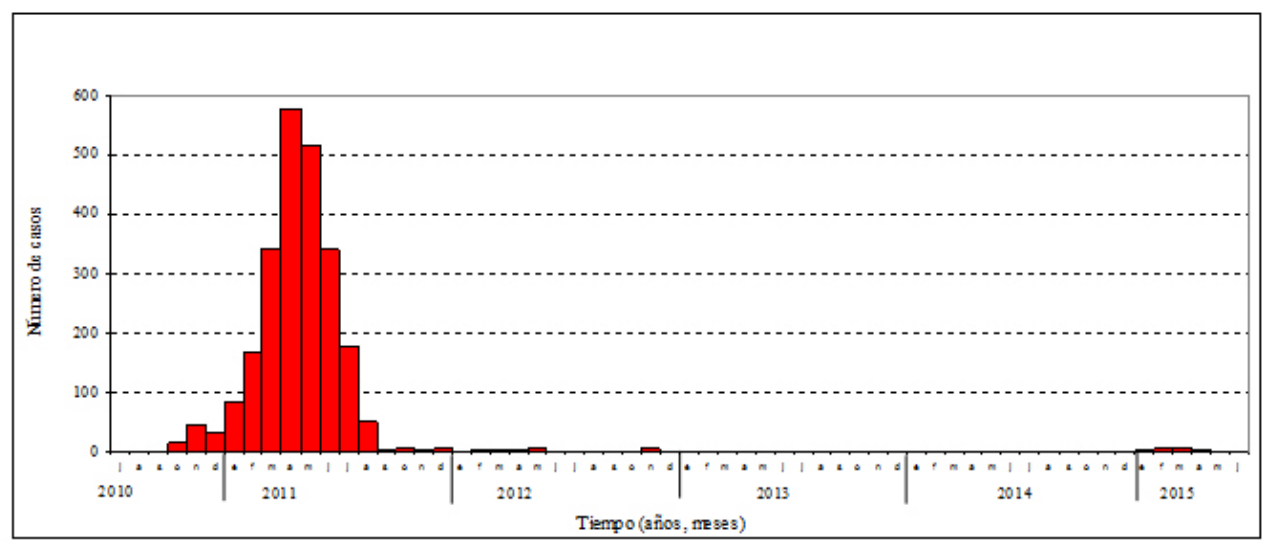

uno se confirmó por laboratorio calificándose como caso importado (de Irlanda) y otro quedó como sospechoso, ya que cumplía la definición clínica de caso pero no se tomaron muestras para su estudio. Durante el primer semestre del 2015 hubo un pequeño brote con 13 casos confirmados y 2 casos sospechosos.

BROTE DE 2010-2011 EN GRANADA ${ }^{12,13}$

Incidencia. Durante el periodo epidémico se registraron 308 casos, lo que supuso una incidencia de 33,55 por 100.000 habitantes, la mayor registrada en Granada en los últimos 20 años. Se confirmaron por laboratorio o por vínculo epidemiológico 287 (93,20\%) casos, y $21(6,80 \%)$ se consideraron compatibles por haber presentado criterios clínicos, aunque no fueron confirmados. Se descartaron 64 sospechas.

Persona. Del total de casos fueron varones 167 (54,22\%), lo que supuso un índice de masculinidad de 1,18 .

La máxima incidencia se registró en niños menores de 2 años con 96 (31,16\%) casos declarados (figura 2), lo que supuso una tasa de incidencia de 519,20 por 100.000 habitantes. Descendió en los niños de 2 a 4 años $(77,02$ por 100.000 habitantes) y de 5 a 14 , para aumentar en el grupo de 15 a 19 años y alcanzar un pequeño pico de elevada incidencia en adultos jóvenes de 20 a 24 años (65 por 100.000 habitantes). Hubo 23 mayores de 34 años de los que 6 fueron mayores de 39 años (1,94\% del total). La mediana de edad fue de 15 años.

Los casos registrados durante la primera parte del brote en Granada capital presentaron un patrón de edad algo diferente al del resto de la provincia, con una mayor incidencia en el grupo de edad 16 meses a 4 años y menor en sujetos de 15 a 25 años.

La tasa de hospitalización fue del 23,05\% (71), máxima en mayores de 34 años $(47,82 \%)$.

Respecto a los datos de laboratorio, el genotipo circulante fue el B3, subgenotipo B3-1.

Durante el periodo epidémico 2010-11 se pudieron identificar en Granada 33 cadenas de transmisión diferentes (casos con vínculo epidemiológico conocido). En 130 casos se registró la existencia de contacto con un caso entre los días 7 y 21 antes del inicio del exantema. En 71 sujetos el contacto fue un familiar, seguido por 20 contagios en centros 
Figura 2

Tasa de incidencia de sarampión por grupos de edad, Granada 2010-2011

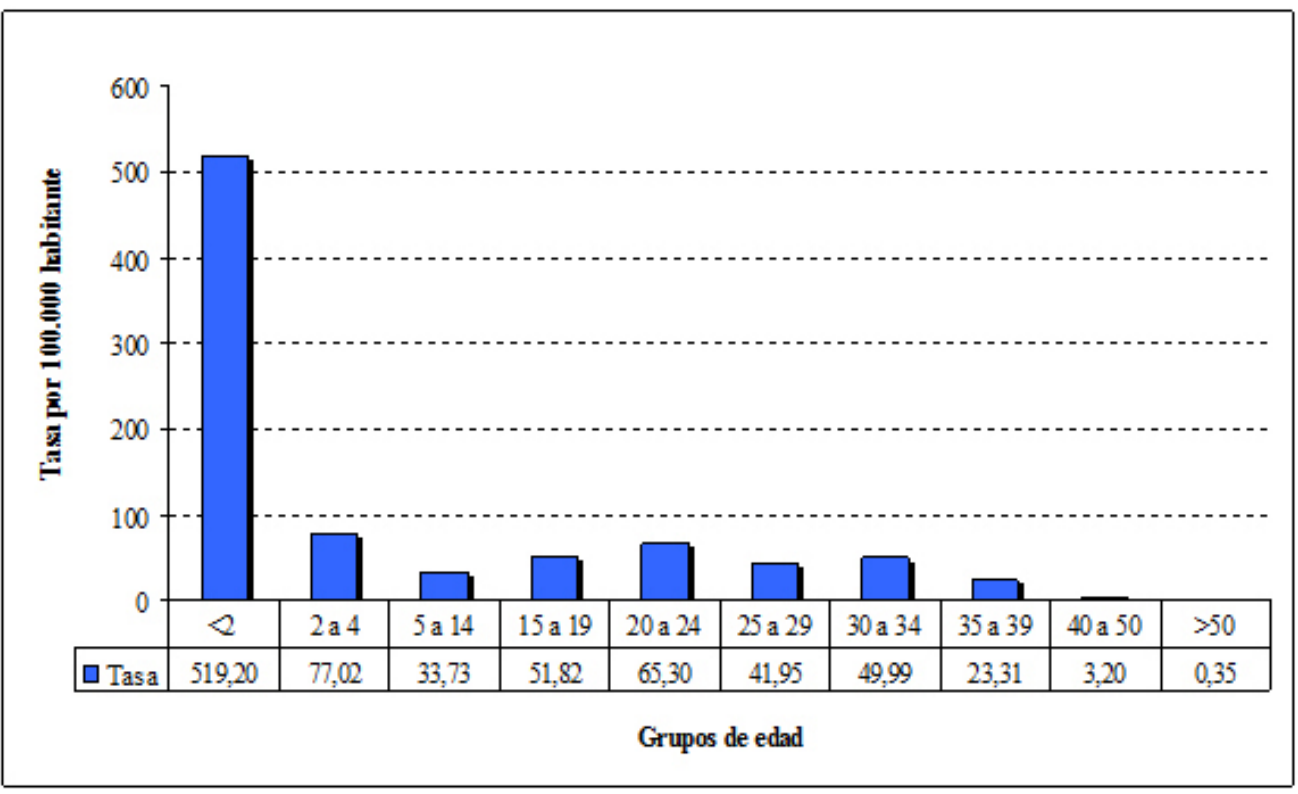

sanitarios (18 en atención especializada, la mayoría de ellos en urgencias, y 2 en atención primaria) y 16 entre compañeros escolares. Se registraron 11 casos en personal sanitario.

La vacunación con TV, documentada o no, se registró en 31 de los 287 (10,80\%) casos confirmados. Se confirmó documentalmente el antecedente de vacunación en 10 (3,48\%), todos ellos con una sola dosis de vacuna. En ninguno constaba haber recibido 2 dosis documentadas de vacuna TV.

Tiempo. El brote transcurrió entre las semanas 41 de 2010 y 34 de 2011. Se pudieron distinguir 3 ondas epidémicas: la primera en las últimas 11 semanas de 2010, la segunda en las primeras 21 semanas de 2011 y la tercera entre la 22 y la 34 de 2011 (figura 3).

Lugar. El brote comenzó en Granada capital, donde muchos de los casos se dieron en familias del Albaicín contrarias a la vacunación. La primera onda epidémica se centró en la ciudad desde donde se fue extendiendo, fundamentalmente a través de los servicios de urgencias de los hospitales, a otras zonas de la provincia. Así, los casos de la segunda onda epidémica, aunque siguieron dándose en Granada capital, aparecieron sobre todo en el distrito metropolitano, del que llegaron a afectarse 30 municipios. Finalmente, el brote se extendió a 4 municipios del Área Sur, dando lugar a la tercera onda epidémica. La máxima incidencia se produjo en el Área Sur, 41,17 por 100.000 habitantes, y en Granada con 42,04 por 100.000 habitantes.

Medidas de intervención. Se aplicaron las medidas de control previstas en el Protocolo de Alerta Epidemiológica por Sarampión del SVEA $^{14}$ que, fundamentalmente, consisten en aislamiento del enfermo durante el periodo de infectividad, localización y seguimiento de los contactos e inmunización de los sujetos susceptibles. Se adelantó la primera dosis de TV en un principio a los 12 meses y luego a los 6 , de forma temporal durante el brote. 
Figura 3

Distribución semanal de casos de sarampión según fecha de declaración, Granada 2010-2011

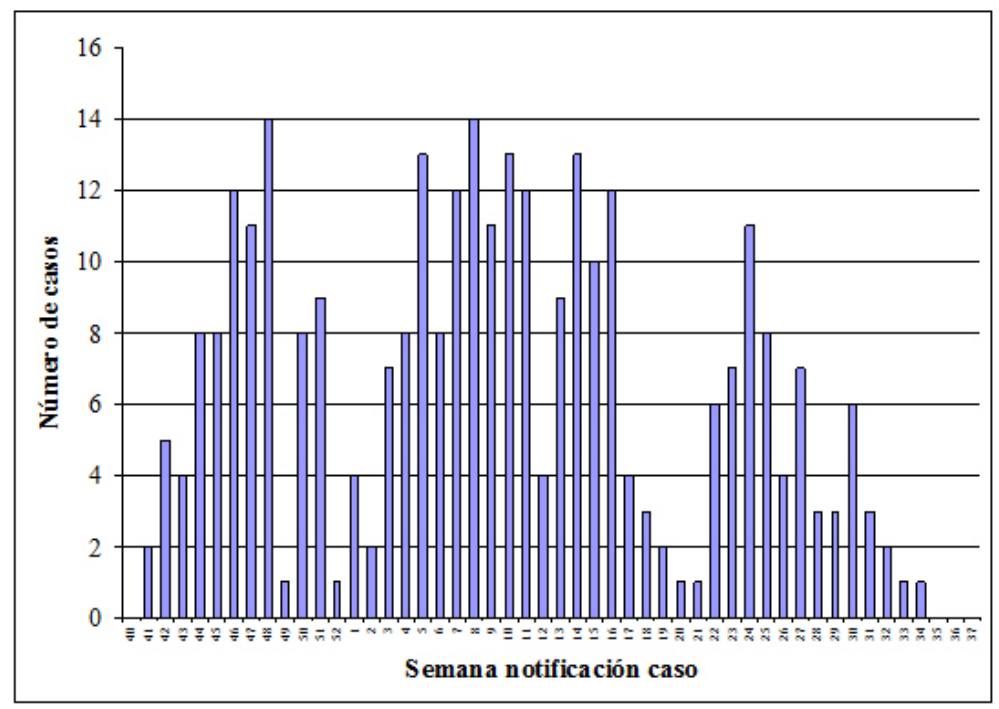

En el ámbito escolar se revisó la situación vacunal de todos los niños y se actualizó el calendario vacunal, administrándose las dosis de TV a quienes no lo tenían completo. La colaboración de algunas familias en la investigación de nuevos casos y en la protección de los contactos fue mala por razones ideológicas (la mayoría rechazaban la vacuna y la inmunoglobulina), sobre todo en 1 de los 4 centros, en el que la cobertura estaba en torno al $60 \%$. Por ello, mediante burofax, a los padres de 79 alumnos se les requirió la vacunación inmediata del niño o la presentación en el colegio de la cartilla de vacunación que documentase su vacunación previa. Ante la ausencia de respuesta de los padres de 35 niños del centro educativo del barrio, la Consejería de Salud solicitó al Juzgado de lo Contencioso Administrativo número 5 de Granada autorización para la adopción de medidas urgentes y necesarias para la Salud Publica, concretamente la vacunación forzosa de los 35 niños escolarizados en dicho CEP cuyos progenitores se habían negado expresamente a que fueran vacunados o no habían entregado los documentos de vacunación solicitados. El titular del Juzgado acordó autorizar la vacunación forzosa y tras visita a los domicilios de los menores quedaron finalmente 9 niños sin vacunar o sin vacunación documentada. Como la proporción de escolares inmunes, bien por vacunación bien por haber pasado la enfermedad, superaba ya el $95 \%$ de los escolares (proporción suficiente para el control del brote) se consideró superada la situación de especial riesgo detectada y por ello no se llevaron adelante más medidas legales.

\section{BROTE DE 2011 EN SEVILLA ${ }^{15,16}$}

Incidencia. Se notificaron 1.760 casos (confirmados y probables) lo que supuso una tasa de incidencia de 21 por 100.000 habitantes. Del total de casos, 827 (47\%) se agruparon en 14 clusters, con una tasa de incidencia superior a 100 por 100.000 habitantes en 5 barrios de la ciudad de Sevilla y 9 municipios cercanos a la ciudad, incluyendo el municipio donde se originó, San Juan de Aznalfarache, donde la tasa de incidencia fue de 489,9 por 100.000 habitantes. En la ciudad de Sevilla, que notificó el $39,9 \%$ de los casos, la tasa de incidencia fue de 99,8 por 100.000 habitantes. 
Persona. El caso índice se notificó el 7 de enero de 2011 en la localidad sevillana de San Juan de Aznalfarache, cercana a la capital. Se trataba de una niña que había estado en contacto durante las navidades con familiares procedentes de Madrid. Seguidamente se produjeron los primeros contagios en el colegio del barrio, donde había una baja cobertura vacunal (tabla 1). El barrio está en una de las zonas denominadas en Andalucía como "zonas con necesidades de transformación social", que son aquellos espacios urbanos claramente delimitados, en cuya población concurren situaciones estructurales de pobreza grave y marginación social, y en los que son significativamente apreciables problemas en vivienda, deterioro urbanístico y déficit de infraestructura, equipamientos y servicios públicos, elevados índices de absentismo y fracaso escolar, altas tasas de desempleo junto a graves carencias formativas profesionales, significativas deficiencias higiénico sanitarias y fenómenos de desintegración social.
El 53,2\% de los casos del brote fueron varones. Afectó a 40 (2,3\%) sanitarios.

El grupo más afectado fue el de los menores de dos años con un $21,9 \%$, lo que supuso una tasa de incidencia de 806,4 por 100.000 (figura 4). El rango de edad fue de 2 semanas a 57 años (media 16,5 años y mediana 16 años).

Respecto a las hospitalizaciones y complicaciones hay que destacar que 294 (16,7\%) pacientes requirieron ingreso hospitalario $\mathrm{y}$ 177 (10,1\%) sufrieron complicaciones.

El genotipo aislado por el laboratorio de referencia fue el D4.

Se pudo obtener información sobre el estado vacunal de 708 enfermos: $26,7 \%$ no habían recibido ninguna dosis de TV y el 13,6\% había recibido al menos una dosis de vacuna TV.

Figura 4

Distribución de casos de sarampión por edad, Sevilla 2011

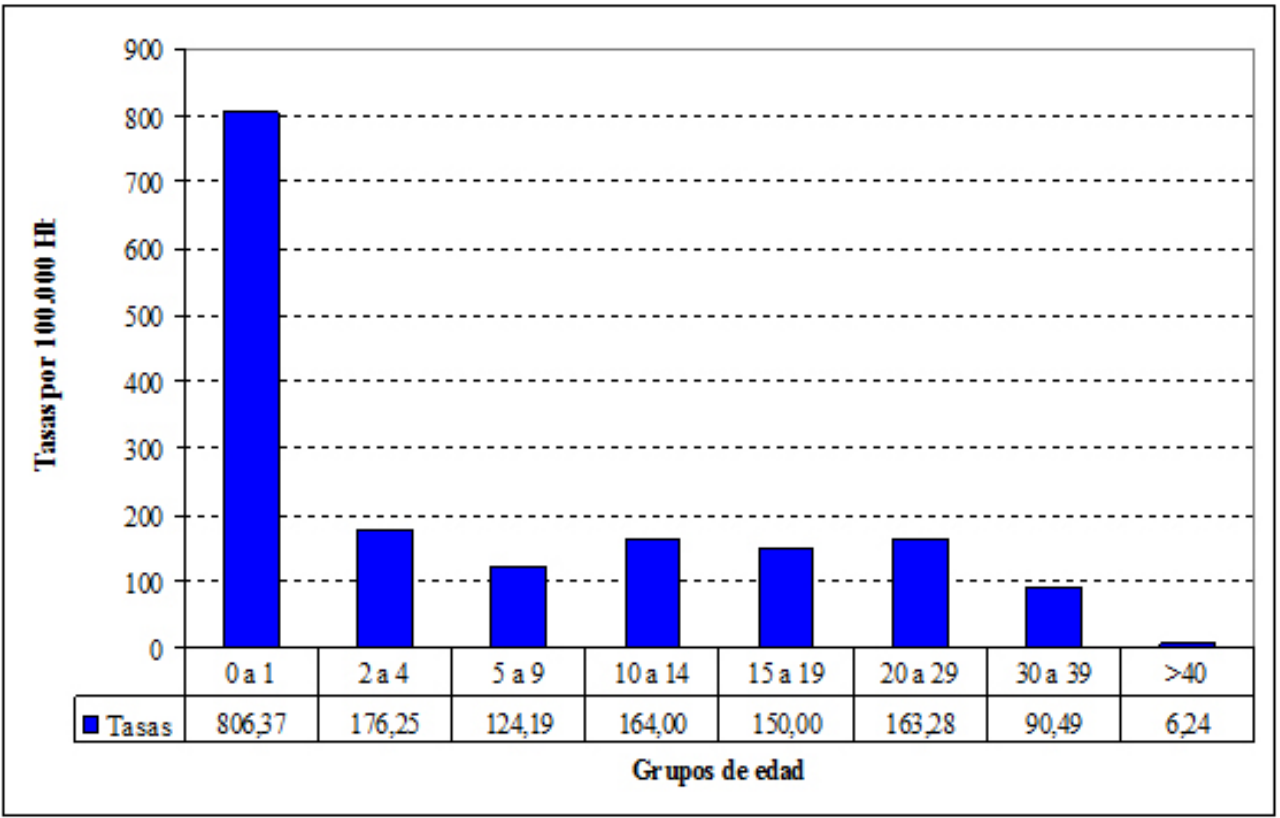


Tiempo. El brote se inició el 31 de diciembre de 2010 y se extendió hasta la semana 35/2011 (figura 5).

En la curva epidémica se observa cómo se fueron presentando las primeras asociaciones de casos por municipio o barriada (en Sevilla capital). Se puede apreciar que la difusión entre los diferentes clusters se estableció manteniendo de forma aproximada el periodo de incubación medio de la enfermedad.

Lugar. Hubo casos en 71 de los 110 municipios de la provincia de Sevilla y en 9 municipios de otras provincias. Sevilla capital fue la localidad más afectada, con el 46,7 \% de los casos.

Respecto a la localización espacial, el brote apareció y se difundió entre barriadas de similares características socioeconómicas (en su mayor parte las denominadas "zonas de transformación social"), con poblaciones también similares y reacias a la vacunación.

Medidas adoptadas. Ante la aparición de casos sospechosos se llevaron a cabo las medidas contempladas en el protocolo de sarampión del SVEA ${ }^{14}$ : aislamiento respiratorio de los casos y vacunación de los contactos susceptibles en las primeras 72 horas.

Tras este brote, como resultado del mismo, se introdujo de forma rutinaria la revisión de las coberturas vacunales dentro del sistema de vigilancia, identificando claramente a los grupos más vulnerables. Esta estrategia se sigue realizando en Atención Primaria, donde trabajan de manera coordinada principalmente epidemiólogos, trabajadores sociales, pediatras y responsables de los puntos de vacunación Desde los servicios centrales del Servicio Andaluz de Salud trimestralmente se envía a cada distrito sanitario la información vacunal de la población infantil (de 0 a 16 años de edad), información extraída de la base de datos de vacunación infantil de Andalucía. Después los listados se dividen por centros de atención primaria o puntos de vacunación y se remiten a cada uno de ellos para

\begin{tabular}{|c|c|c|}
\hline \multicolumn{3}{|c|}{ Tabla 1 } \\
$\begin{array}{c}\text { Cobertura de vacunación de TV } \\
\text { e incidencia de sarampión según edad en } \\
\text { colegio donde ocurrieron los primeros } \\
\text { casos del brote (febrero 2011) }\end{array}$ \\
\hline $\begin{array}{c}\text { Grupos } \\
\text { de edad y sexo }\end{array}$ & $\begin{array}{c}\text { Cobertura } \\
\text { vacunal (\%) }\end{array}$ & $\begin{array}{c}\text { Incidencia } \\
\text { por } 10^{5} \text { habitantes }\end{array}$ \\
\hline Niños de 03 años & 85 & 4 \\
\hline Niños de 04 años & 35 & 20 \\
\hline Niños de 05 años & 80 & 4 \\
\hline Niños de 06 años & 45 & 26 \\
\hline Niños de 07 años & 50 & 28 \\
\hline Niños de 08 años & 55 & 25 \\
\hline Niños de 09 años & 57 & 11 \\
\hline Niños de 10 años & 60 & 5 \\
\hline Niños de 11 años & 37 & 17 \\
\hline
\end{tabular}

su revisión y adopción de las medidas correspondientes (captación activa,...)

Además, a consecuencia de este brote, se introdujeron cambios en el calendario vacunal, adelantando la primera dosis de TV de los 15 meses a los 12 .

\section{BROTE DE 2015 EN GRANADA}

Incidencia. En 2015 se registró en Granada un brote con 15 casos de sarampión, 13 confirmados ( 1 de ellos probablemente postvacunal) y 2 casos compatibles. Esto supuso una incidencia de 1,63 por 100.000 habitantes.

Persona. Del total de casos, el $60 \%$ se dio en varones.

La edad media fue de 15,9 años, con un $46,7 \%$ en menores de 5 años. No hubo casos entre 5 y 14 años. El 33\% fueron mayores de 24 años. La confirmación de laboratorio se realizó por serología positiva en 12 personas, en 4 de las cuales además se obtuvo una PCR positiva. En una la confirmación se realizó solo por la PCR. El genotipo de sarampión de los 5 casos positivos por PCR y/o cultivo fue D8 y el tipo de variante fue MVs/RostovonDon.RUS/47.13/2-variant. 
Figura 5

Distribución de casos según fecha de declaración y municipio, Sevilla 2011

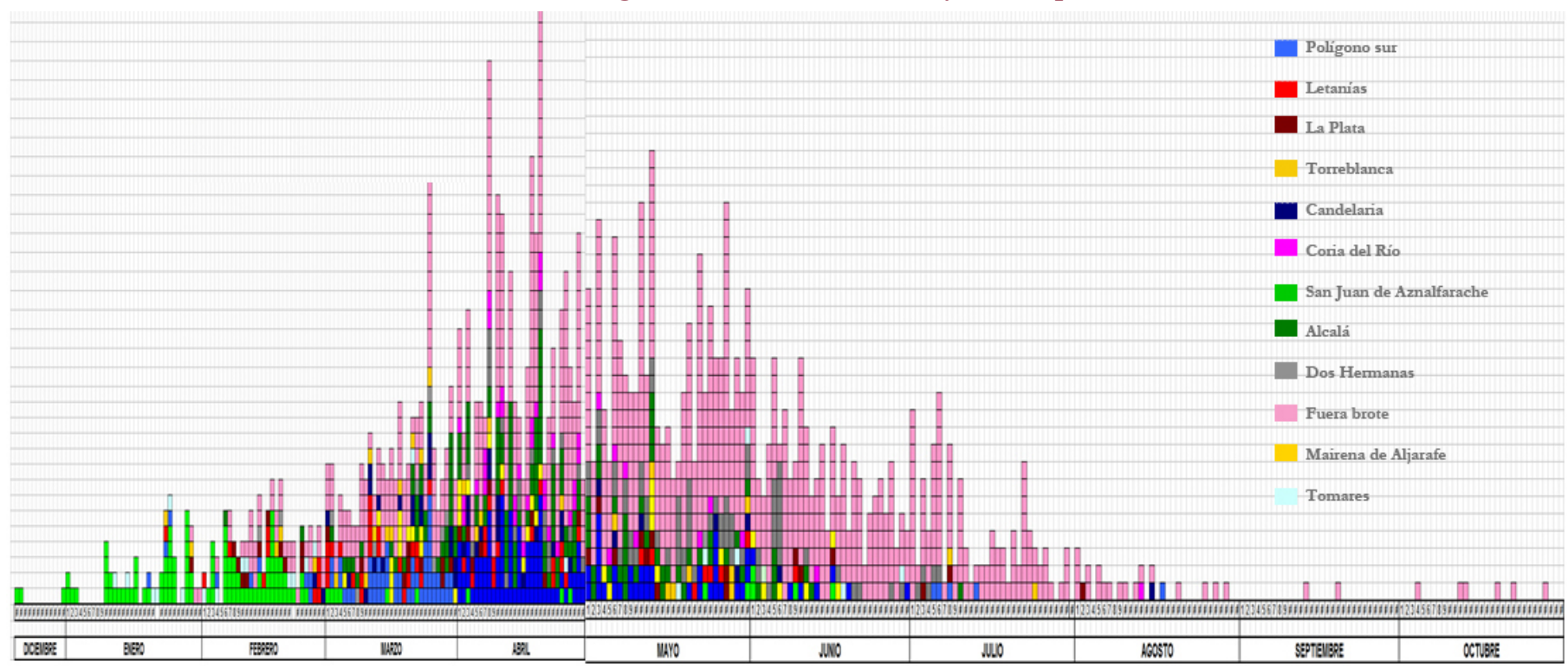

Figura 6

Distribución de casos según fecha de declaración y municipio, Granada 2015

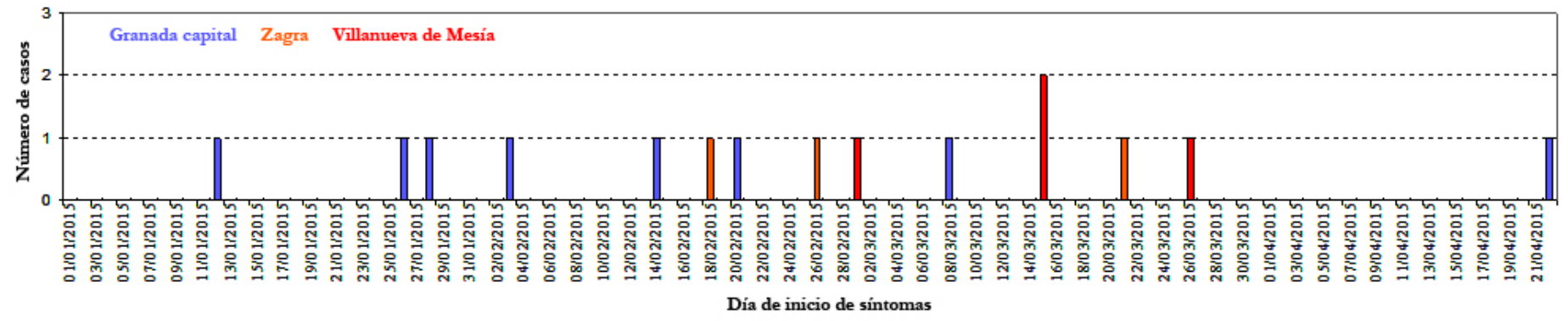


En cuanto al estado vacunal, de los 7 casos infantiles 3 fueron menores de 12 meses, 2 no estaban vacunados y los otros 2 presentaron antecedente de 1 dosis de vacuna. Uno de ellos pudo ser un caso postvacunal y el otro se quedó como caso sospechoso, ya que no se pudo confirmar/descartar el diagnóstico por falta de serología. En los adultos no se constató antecedente de vacunación en ninguno de ellos.

Tiempo. El primer caso comenzó con síntomas en la semana 2 de 2015 y el último en la semana 17. En el tiempo intermedio hubo un goteo de casos, respetando los periodos de incubación (figura 6).

Lugar. El brote comenzó con 2 personas adultas que llegaron a Granada capital desde países de Europa en periodo de incubación.

En el municipio de Granada se registraron en total 6 casos y la enfermedad se extendió a otros 5 municipios, todos del distrito metropolitano, aunque solo en 2 hubo transmisión: Zagra (3 casos) y Villanueva de Mesía (3 casos).

El brote se inició, por tanto, con 2 casos importados y la transmisión se realizó fundamentalmente por contacto en las urgencias de los centros sanitarios y de forma intrafamiliar.

No se produjo ningún caso entre personal sanitario.

Medidas adoptadas. Se adoptaron las medidas de control recogidas en el protocolo de

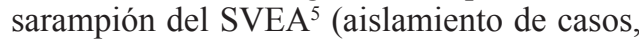
identificación e inmunización de contactos).

Estas medidas se implementaron en los escasos centros escolares afectados y en los centros sanitarios, además de en centros laborales en el caso de los adultos. Se trabajó en la identificación de posibles zonas con deficientes coberturas y se hizo especial hincapié en la información y sensibilización a trabajadores de centros sanitarios (comunicación a todas las unidades de atención primaria y especializada y realización y difusión de cartelería informativa para los servicios de urgencias). La actuación fue muy precoz en la mayor parte de los casos, lo que, en gran medida limitó la difusión de la enfermedad y facilitó el control del brote.

\section{DISCUSIÓN}

A pesar del alto nivel de cobertura vacunal de la Comunidad Autónoma de Andalucía, el virus del sarampión provocó tres brotes durante el período 2010-2015, todos ellos con caso índice importado y con tres genotipos diferentes (B3, D4, D8) que coinciden con los circulantes en los diferentes años en Europa. Los principales grupos de edad afectados fueron bebés no vacunados de 6 a 15 meses de edad y población joven de 20 a 34 años. La mejora de la cobertura de vacunación, tanto infantil como en adultos jóvenes, es clave en la prevención y control del virus del sarampión junto con la vacunación de grupos de población que suelen presentar baja cobertura vacunal. El principal brote acaecido (Sevilla 2011) comenzó en sujetos no vacunados del colectivo romaní y se extendió desde su inicio por zonas denominadas "con necesidades de transformación social”. Este brote estuvo relacionado con grupos sociales en los que había población infantil no vacunada y elevado número de sujetos susceptibles. Su gran movilidad geográfica y la estrecha relación que se mantiene entre familiares que viven en diferentes lugares facilitó la propagación a otros municipios. Se extendió principalmente entre lactantes y entre las personas de la "bolsa histórica de susceptibles" (no vacunadas por haber nacido antes del inicio de la vacunacióny que no han pasado la enfermedad porque la vacunación interrumpió rápidamente la circulación del virus) en poblaciones vacunadas. Es fundamental, como propone la $\mathrm{OMS}^{17}$, mantener la cobertura vacunal elevada (igual o superior al 95\%) con las dos dosis de vacuna frente al sarampión que tiene actualmente Andalucía, prestando especial atención a los grupos de población que posiblemente presenten baja cobertura vacunal 
(grupos étnicos minoritarios, grupos que rechacen la vacunación por motivos religiosos u otros) y ofrecer una segunda oportunidad de inmunización frente al sarampión mediante actividades suplementarias de inmunización en grupos de población susceptibles (estudiantes universitarios, trabajadores en centros sanitarios).

Otro punto a destacar de los brotes epidémicos fue la transmisión del virus en centros escolares y sanitarios, donde es preciso actuar con rapidez y contundencia. La transmisión nosocomial del sarampión se dio en los tres brotes descritos. El aislamiento rápido ante la sospecha de un caso de sarampión que demanda asistencia y el mantenimiento de altas coberturas vacunales en los trabajadores sanitarios son las dos estrategias fundamentales para prevenir la transmisión del virus en estos centros, especialmente en los servicios de urgencias, donde pueden acudir casos durante el período de transmisibilidad que actúen como fuente de infección, tanto para los trabajadores no inmunizados como para el público que acuda y no esté inmunizado. La OMS propone ${ }^{17}$, como otra de las estrategias para la eliminación del sarampión, mejorar la disponibilidad de la información dirigida a los trabajadores sanitarios sobre los beneficios y riesgos asociados a la vacunación frente a sarampión, como se hizo en el último brote acaecido en Granada durante 2015.

En conclusión, en el contexto actual, con un incremento del número de casos en muchos países europeos y americanos, es necesario reforzar las medidas de prevención y control de la transmisión del virus del sarampión. Las estrategias de prevención, como se ha aprendido en los brotes del 2010 y 2011, deben estar encaminadas al mantenimiento de una alta cobertura vacunal infantil para las dos dosis, a la inmunización de grupos de población susceptible y al mantenimiento de un sistema de vigilancia capaz de detectar precozmente los casos para poder controlar la difusión del virus en la población. Es fundamental indagar a tiempo dónde, cuándo y en qué grupos de población bajan las coberturas de vacunación. Sólo el seguimiento de la evolución de las coberturas a nivel local permite evitar la formación de bolsas de individuos susceptibles. El brote de 2015 demostró que con ello y con un exhaustivo control de los contactos se puede llegar a cortar rápido la transmisión.

Así, se debe continuar trabajando para conseguir los objetivos que establece la OMS en materia de vigilancia epidemiológica ${ }^{17}$, entre los que destacan detectar e investigar todos los casos aislados y los clusters, con el fin de garantizar un manejo adecuado de casos y contactos, investigar la cadena de transmisión (casos importados, huéspedes susceptibles), evaluar su duración (tamaño, duración de las agrupaciones de casos), identificar grupos de población susceptible y garantizar una respuesta de salud pública rápida y apropiada. Además es fundamental monitorizar la incidencia de la enfermedad y la circulación del virus, con el fin de evaluar el progreso hacia los objetivos de eliminación, identificar cambios de las características epidemiológicas de la enfermedad, evaluar la circulación de los genotipos virales y proporcionar información para la planificación y evaluación de programas preventivos.

\section{AGRADECIMIENTOS}

A todos los epidemiólogos locales y resto de profesionales sanitarios (médicos preventivistas, pediatras, trabajadores sociales, enfermeras,...) que han trabajado en los distintos brotes y sin la labor de los cuales no hubiera sido posible el control de los mismos. Y a todos los profesionales de los puntos de vacunación que trabajan diariamente para mantener las coberturas que posee Andalucía.

\section{BIBLIOGRAFÍA}

1. Boletín Oficial de la Junta de Andalucía. Decreto 66/1996, de 13 de febrero, por el que se constituye, en la Comunidad Autónoma de Andalucía, el Sistema de Vigilancia Epidemiológica y se determinan normas sobre el mismo. BOJA núm 35 de 19-03-1996. 
2. Boletín Oficial de la Junta de Andalucía. Orden de 19 de diciembre de 1996, por la que se desarrolla el Sistema de Vigilancia Epidemiológica en la Comunidad Autónoma de Andalucía y se establece la relación de enfermedades de declaración obligatoria. BOJA núm 4 de 09-01-1997.

3. Boletín Oficial de la Junta de Andalucía. Orden de 11 de diciembre de 2008, por la que se modifica la Orden de 19 de diciembre de 1996, por la que se desarrolla el Sistema de Vigilancia Epidemiológicaen la Comunidad Autónoma de Andalucía y se establece la relación de enfermedades de declaración obligatoria. BOJA núm 4 de de 08-01-2009.

4. Boletín Oficial de la Junta de Andalucía Orden de 25 de junio de 2007, por la que se establecen los criterios de aplicación y valoración del complemento de productividad mediante método directo al personal funcionario de la Consejería que desempeñen las funciones de la Red de Alerta del Sistema Sanitario Público de Andalucía, fuera del horario laboral. BOJA núm 140 de17-07-2007.

5. Protocolo de Alerta Epidemiológica por Sarampión. Sevilla: Consejería de Salud, Junta de Andalucía; 2014. [citado el 20-6-2015]. Disponible en: http://www.csalud. junta-andalucia.es/ salud/export/sites/csalud/galerias/ documentos/p_4_p_1_ vigilancia_de_la_salud/sarampion_2014.pdf

6. Plan de Acción para la Eliminación del Sarampión en Andalucía. Sevilla: Dirección General de Salud Pública y Participación, Consejería de Salud, Junta de Andalucía; 2001. [citado el 20-6-2015]. Disponible en: http:// www. hvn.es/servicios_asistenciales/microbiologia_-_ servicio/ ficheros/plansarampion_andalucia.pdf

7. Plan de acción para la eliminación del sarampión en España. Madrid: Centro Nacional de Epidemiología; 2000. [citado el 20-6-2015]. Disponible en: http://www.isciii. es/ISCIII/es/contenidos/fd-servicios-cientifico-tecnicos/ fd-vigilanciasalertas/fd-enfermedades/fd-enfermedadespreveniblesvacunacion/PLANSARAMPION.pdf

8. Eliminating measles and rubella and preventing congenital rubella infection: WHO European Region Strategic Plan 2005-2010. Copenhagen: World Health Organization Regional Office for Europe; 2005. [citado el 20 Junio 2015]. Disponible en: http://www.euro.who.int/InformationSources/Publications/Catalogue/20051123_1

9. Calendario de Vacunación Infantil, 2015. Sevilla: Consejería de Salud, Junta de Andalucía. [citado el 226-2015]. Disponible en: http://www.juntadeandalucia.es/ salud/ZHD/vacunas2012/

10. Cobertura de vacunación. Datos estadísticos. Madrid: Ministerio de Sanidad, Servicios Sociales e Igualdad. [citado el 30-6-2015]. Disponible en: http://www.msssi. gob.es/profesionales/saludPublica/prevPromocion/vacunaciones/coberturas.htm\#tercero

11. World Health Organization. Global measles and rubella strategic plan: 2012-2020. Geneva: WHO; 2012.
12. López Hernández B, Laguna Sorinas J, Marín Rodríguez I, Gallardo García V, Pérez Morilla E, Mayoral Cortés JM. Spotlight on measles 2010: An ongoing outbreak of measles in an unvaccinated population in Granada, Spain, October to November 2010. Euro Surveill. 2010; 15(50): pii=19746

13. Navarro E, Mochón MM, Galicia MD, Marín I, Laguna J. Study of a measles outbreak in Granada with reventive measures applied by the courts, Spain, 2010 to 2011. Euro Surveill. 2013; 18(43): pii $=20612$

14. Protocolo de Alerta Epidemiológica por Sarampión. Sevilla: Consejería de Salud, Junta de Andalucía; 2008. [citado el 23-6-2015]. Disponible en: http://www. juntadeandalucia.es/salud/export/sites/csalud/galerias/ documentos/p_4_p_1_vigilancia_de_la_salud/Protocolos_actuacion/sarampion.pdf

15. Mayoral Cortes JM, Perez Morilla E, Gallardo Garcia V, Navarro Marí, JM, Perez Ruiz M, Hermosilla R, Diaz-Borrego J, Rodriguez Romero E, Ruiz Fernandez J. Measles outbreak in Andalusia, Spain, January to August 2011. Euro Surveill. 2012; 17 (42): pii $=20300$

16. Luna Sánchez A, Rodríguez Benjumeda LM y Ortega Sánchez PC. Análisis de un brote de sarampión en una barriada de la provincia de Sevilla, España. Rev Esp Salud Pública 2013; 87:257-266

17. Surveillance Guidelines for Measles, Rubella and Congenital Rubella Syndrome in the WHO European Region. Copenhagen, WHO. Regional Office for Europe. Update December 2012. [citado el 2-7-2015]. Disponible en: http://www.euro.who.int/_data/assets/ pdf_file/0018/79020/e93035-2013.pdf 